Supplementary Information for

\title{
How stable and powerful can metal cyclo-pentazolate salts achieve? An answer through theoretical crystal design
}

\author{
Chuang Yao ${ }^{a}$, Yezi Yang ${ }^{a}, Y i Y u^{b}$, Chang Q Sun ${ }^{a}$, Xin-Xin Wang ${ }^{c,}{ }^{*}, H u i$ Li $^{d}$, Haijian Li ${ }^{d}$, Lei \\ Zhang ${ }^{b, c, *}$ \\ ${ }^{a}$ Key Laboratory of Extraordinary Bond Engineering and Advance Materials Technology \\ (EBEAM) of Chongqing, School of Materials Science and Engineering, Yangtze Normal \\ University, Chongqing 408100, China; \\ ${ }^{b}$ CAEP Software Center for High Performance Numerical Simulation, Beijing, 100088, China; \\ ${ }^{c}$ Laboratory of Computational Physics, Institute of Applied Physics and Computational \\ Mathematics, Beijing, 100088, China. \\ ${ }^{d}$ Science and Technology on Combustion and Explosion Laboratory, Xi'an Modern Chemistry \\ Research Institute, Xi'an 710065, China. \\ "Correspondence author: X.W. (xxwang14b@alum.imr.ac.cn) and L.Z. (zhang_lei@iapcm.ac.cn)
}

The supplementary information contains cif files of the first three lowest energy crystal structures for each of the four metal cyclo-pentazolate salts.

$\mathbf{N a N}_{5}$ : NaN5-lowest-energy.cif, NaN5-second-lowest-energy.cif, NaN5-third-lowest-energy.cif;

$\mathbf{M g}\left(\mathbf{N}_{5}\right)_{2}:$ MgN5_2-lowest-energy.cif, MgN5_2-second-lowest-energy.cif, MgN5_2-third-lowestenergy.cif;

$\operatorname{Co}\left(\mathbf{N}_{5}\right)_{2}$ : CoN5_2-lowest-energy.cif, CoN5_2-second-lowest-energy.cif, CoN5_2-third-lowestenergy.cif;

$\operatorname{Mn}\left(\mathbf{N}_{5}\right)_{2}$ : MnN5_2-lowest-energy.cif, MnN5_2-second-lowest-energy.cif, and MnN5_2-thirdlowest-energy.cif 